Doug Geisler, Eva K. Grebel, and Dante Minniti, eds.

\title{
Bulge Globular Clusters
}

\author{
B. Barbuy
}

University of São Paulo, IAG, C.P. 3386, São Paulo 01060-970, Brazil

S. Ortolani

University of Padova, Vicolo dell'Osservatorio 5, I-35122 Padova, Italy

E. Bica

University of Rio Grande do Sul, C.P. 15051, Porto Alegre 91501-970, Brazil

\begin{abstract}
The properties of the globular clusters located within $20^{\circ} \times 20^{\circ}$ of the Galactic Center are discussed. In particular their spatial distribution, metallicities and ages are presented and discussed in the context of different scenarios of bulge formation.
\end{abstract}

\section{Introduction}

Bulges of spirals seem to be of two types, the more massive bulges of $\mathrm{Sa}$ and $\mathrm{Sb}$ spirals, and bulges of Sc galaxies, in the latter case sometimes even absent. The Milky Way is an Sbc type galaxy. Two main competing scenarios for the formation of bulges of spirals are the inside-out model (e.g., Larson 1990) producing old bulges, and the dynamical evolution of bars (e.g., Raha et al. 1991; Friedli \& Benz 1995; Norman et al. 1996). Gadotti \& dos Anjos (2001) find that 25\% of bulges of Sbc galaxies present flat colour gradients, which may be due to the action of bars.

The Galactic bulge allows us to study and disentangle the different stellar populations it contains, as well as to compare its overall properties to those of bulges of external galaxies.

Globular clusters can be used as tracers of the spatial distribution, metallicity and ages of the bulge's old stellar population. Among the 150 known globular clusters of the Milky Way, most are projected against the Galactic center.

\section{The bulge globular cluster system}

Among the 150 known globular clusters of the Milky Way, 74 are seen in projection within $20^{\circ} \times 20^{\circ}$ of the Galactic Center, and among these 60 are within $4 \mathrm{kpc}$ of the Center. They form a flattened system, extending to $4.5 \mathrm{kpc}$ from the Sun. Most metal-rich clusters, defined to have $[\mathrm{Fe} / \mathrm{H}]>-1.0 \mathrm{dex}$, belong to this bulge sample. The bulge shows a mean rotation of $150 \mathrm{~km} \mathrm{~s}^{-1}$, whereas the halo rotates on average with $50 \mathrm{~km} \mathrm{~s}^{-1}$. 
The importance of studying the bulge clusters stems from two main goals:

(a) They trace the structure and metallicity distribution of the Galaxy. Their study may lead us to distinguish between the two main scenarios for bulge formation, which are (i) the classical scenario of inside-out galaxy formation, in which the bulge forms in the first $\sim 10^{8}$ years in free fall $\left(\mathrm{t}_{\mathrm{ff}} \alpha(\mathrm{G} \rho)^{-1 / 2}\right.$, (ii) dynamical evolution of bars, with transfer of disk gas and stars to the Galaxy center.

(b) The detailed abundance analysis of individual stars, and their integrated spectra can be used as templates for the study of composite stellar populations of ellipticals and bulges of spirals (Bica 1988).

\subsection{Recently detected globular clusters within $20^{\circ} \times 20^{\circ}$ of the Galac- tic Center}

Note that the list of globular clusters in the Galaxy has been changing with new objects added in the last years such as:

Lyngå 7 (Ortolani et al. 1993; Tavarez \& Friel 1995), Pyxis (Weinberger 1995; Da Costa 1995; Irwin et al. 1995; Sarajedini \& Geisler 1996) and IC 1257 (Harris et al. 1997). More recently Hurt et al. (2000) identified two new globular clusters from the 2MASS Survey, 2MASS-GC01 $\left(l=10^{\circ} .47, b=0 .{ }^{\circ} 10\right)$ and 2MASS-GC02 $(l=9.78, b=0.62)$, and Ortolani et al. (2000) have shown that the colour-magnitude diagram (CMD) of ESO 280-SC06, previously classified as an open cluster, is instead typical of a globular cluster, and should be added as the $150^{\text {th }}$ to the list of globular clusters of Harris (1996), together with the two 2MASS ones.

In the very central parts of the Galaxy, where extinction is the highest, there probably are globular clusters that may be detectable only in infrared colours. In fact, based on the 2MASS survey, Dutra \& Bica (2000) provide a list of about 58 candidates for central star clusters in the Galaxy. Some of them may turn out to be globular clusters.

A number of blue HB metal-poor globular clusters are contained in the bulge sample, such as Terzan 4, Terzan 9, HP 1, NGC 6139, NGC 6453, NGC 6522. Barbuy et al. (1999) presented a list of bulge clusters with the identification of red, blue or intermediate horizontal branches. The metal-poor globular clusters located in the bulge could be halo visitors, or true bulge members, in which case they might be the oldest objects in the Galaxy. HST CMDs for a few metalpoor globular clusters located in the $20^{\circ} \times 20^{\circ}$ region were presented by Hesser (this meeting), such as NGC 6287, which seem to have a similar age to that of halo clusters. It would be important to obtain CMDs for all of these metal-poor clusters, in order to know if all of them may be halo clusters, or if a few might be extremely old bulge clusters, as suggested by van den Bergh (1993).

\subsection{Metallicity distribution}

The metallicity distribution of the high resolution spectroscopic analysis of field stars of the Baade Window by McWilliam \& Rich (1994) and that of the 17 known globular clusters within $5^{\circ}$ of the Galactic center by Barbuy et al. (1998), are essentially similar, and should roughly represent the bulge metallicity distribution. 


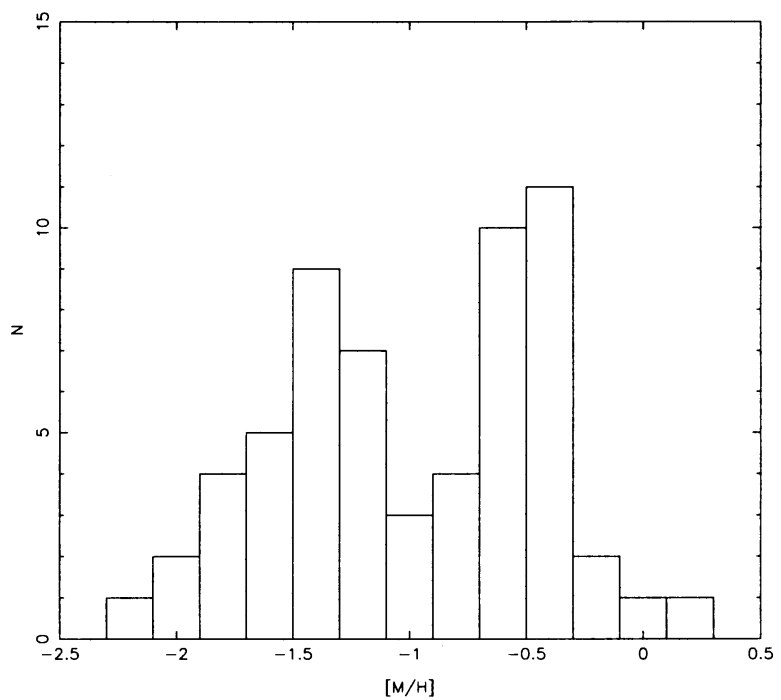

Figure 1. Histogram of metallicities of 60 globular clusters within $\mathrm{R}_{\mathrm{GC}}<4 \mathrm{kpc}$ and $20^{\circ} \times 20^{\circ}$ of the Galactic center, where metallicity values are adopted from Harris (1996).

The metallicity distribution of the 60 globular clusters within $\mathrm{R}_{\mathrm{GC}}<4$ kpc and $20^{\circ} \times 20^{\circ}$ of the Galactic center, based on metallicity values reported in Harris (1996, updated in the website http://physun.physics.mcmaster.ca/Globular.html), is shown in Fig. 1. The histogram of Fig. 1 appears to show bulge and halo populations, and it is interesting to compare this figure to Fig. 8 of Côté (1999) applied to 133 Galactic globular clusters. Here a metal-rich population of $[\mathrm{M} / \mathrm{H}] \sim-0.7$ dex similar to that of Fig. 1 is seen, whereas the "halo" peak at $[\mathrm{M} / \mathrm{H}] \sim-1.5$ dex is much more populated. Côté (1999) defines a third population of intermediate metallicity clusters at $[\mathrm{M} / \mathrm{H}] \sim-1.1$.

\section{An old bulge or a bar}

"Do the globular clusters around the Galactic center belong to the bulge or to the thick disk?" Minniti (1995) has shown that the spatial distribution of the inner metal-rich clusters has a steep density law $\rho_{\text {bulge }} \sim \mathrm{r}^{-\mathrm{m}}$ with $\mathrm{m}=3.65$ to 4.2 , consistent with the field population in the inner $3 \mathrm{kpc}$ (Terndrup 1988). The bulge field giants as well as the bulge clusters are very concentrated, and this is more consistent with a bulge than with a thick disk distribution. In his Fig. 4 Minniti (1995) shows also that velocities for the metal-rich clusters within $30^{\circ}$ of the Galactic center fit well a bulge rotation as a solid body as given in Minniti (1994). The same conclusion is drawn by Côté (1999), who 
finds that the rotation curve of metal-rich globular clusters follows that of inner bulge field stars, and that there is agreement also with the inner metal-poor and intermediate metallicity globulars.

"Is the bulge old or is it the result of the dynamical evolution of a bar?" This question is still open. Côté (1999) plots the longitude - velocity diagram in the direction of the Galactic center, where the $\mathrm{H}$ I data contours (Burton \& Liszt 1983) seem to coincide with the location of metal-rich globular clusters.

The measure of the proper motion of NGC 6553 by Zoccali et al. (2001) based on two epoch HST data, gives $(\Pi, \Theta, W)=(-3.5,230,-3) \mathrm{km} \mathrm{s}^{-1}$, therefore its rotational velocity is consistent with both the rotation of the bulge at $2.7 \mathrm{kpc}$ (Minniti 1995) and the disk rotation at the same distance (Amaral et al. 1996). From this evidence, NGC 6553 could belong to the disk population, or alternately, the old bulge population might share similar kinematical properties with the disk population at that particular position in the Galaxy. Indeed, this cluster is very similar to NGC 6528, located in Baade's Window, with a radial velocity of $\mathrm{v}_{\mathrm{r}} \approx 215 \mathrm{~km} \mathrm{~s}^{-1}$, and an old age. In fact, the old age of NGC 6553 and NGC 6528 was first pointed out by Ortolani et al. (1995), based on optical CMDS with HST data (see Fig. 2), and the old age of NGC 6528 is now confirmed with HST-NICMOS data (Ortolani et al. 2001; Ortolani 2002, this meeting), by comparison of J,H diagrams with isochrones by Cassisi et al. $(1997,1998)$ and more recent unpublished calculations.

\section{Abundances of individual stars in the Galactic bulge}

Very few high resolution abundance analyses of bulge stars are available. Analyses of bulge field stars of Baade's Window were presented by McWilliam \& Rich (1994) and Rich \& McWilliam (2000), where they find a metallicity range of $-1.6<[\mathrm{Fe} / \mathrm{H}]<+0.55$ dex and overabundances of the $\mathrm{Mg}$ and $\mathrm{Ti}$, together with solar abundances of $\mathrm{Ca}$ and $\mathrm{Si}$ (also $\alpha$ elements). An oxygen overabundance is reported in Rich \& McWilliam (2000).

Individual stars of bulge clusters were analysed based on high resolution spectra only for NGC 6553: Barbuy et al. (1999) analysed two red giants and Cohen et al. (1999) analysed horizontal branch (HB) stars. The results are summarized in Table 1 . There appears a discrepancy between the $[\mathrm{Fe} / \mathrm{H}]$ value of Barbuy et al. $([\mathrm{Fe} / \mathrm{H}] \approx-0.55)$ and Cohen et al. $([\mathrm{Fe} / \mathrm{H}] \approx-0.17 \mathrm{dex})$. It is interesting to note that Coelho et al. (2001) analysed low resolution spectra of stars from the red giant to HB stars of NGC 6528 and NGC 6553, and found as well a difference in metallicity between red giants and HB stars. This might be due to model atmosphere problems and has to be further investigated. On the other hand, the overall metallicity of NGC 6553 between Barbuy et al. $\left(\left[\mathrm{Z} / \mathrm{Z}_{\odot}\right]\right.$ $\approx-0.25$, adopting the same excesses of the $\alpha$ elements: $\mathrm{O}, \mathrm{Ne}, \mathrm{Mg}, \mathrm{Si}, \mathrm{S}, \mathrm{Ar}$, $\mathrm{Ca}, \mathrm{Ti})$ and Cohen et al. $\left[\mathrm{Z} / \mathrm{Z}_{\odot}\right] \approx+0.16$ if $[\mathrm{O} / \mathrm{Fe}]=+0.3$, or $\left(\left[\mathrm{Z} / \mathrm{Z}_{\odot}\right] \approx-0.1\right.$ if $[\mathrm{O} / \mathrm{Fe}]=0.0-$ these two oxygen values are considered since they used the $\mathrm{O}$ I triplet lines to derive oxygen abundances, which lines tend to give overestimated values), are compatible within errors. Some examples of combinations between $[\mathrm{Fe} / \mathrm{H}],[\mathrm{O} / \mathrm{Fe}],[\alpha / \mathrm{Fe}]$ and resulting $\left[\mathrm{Z} / \mathrm{Z}_{\odot}\right]$ values are given in Table 2 . It is 


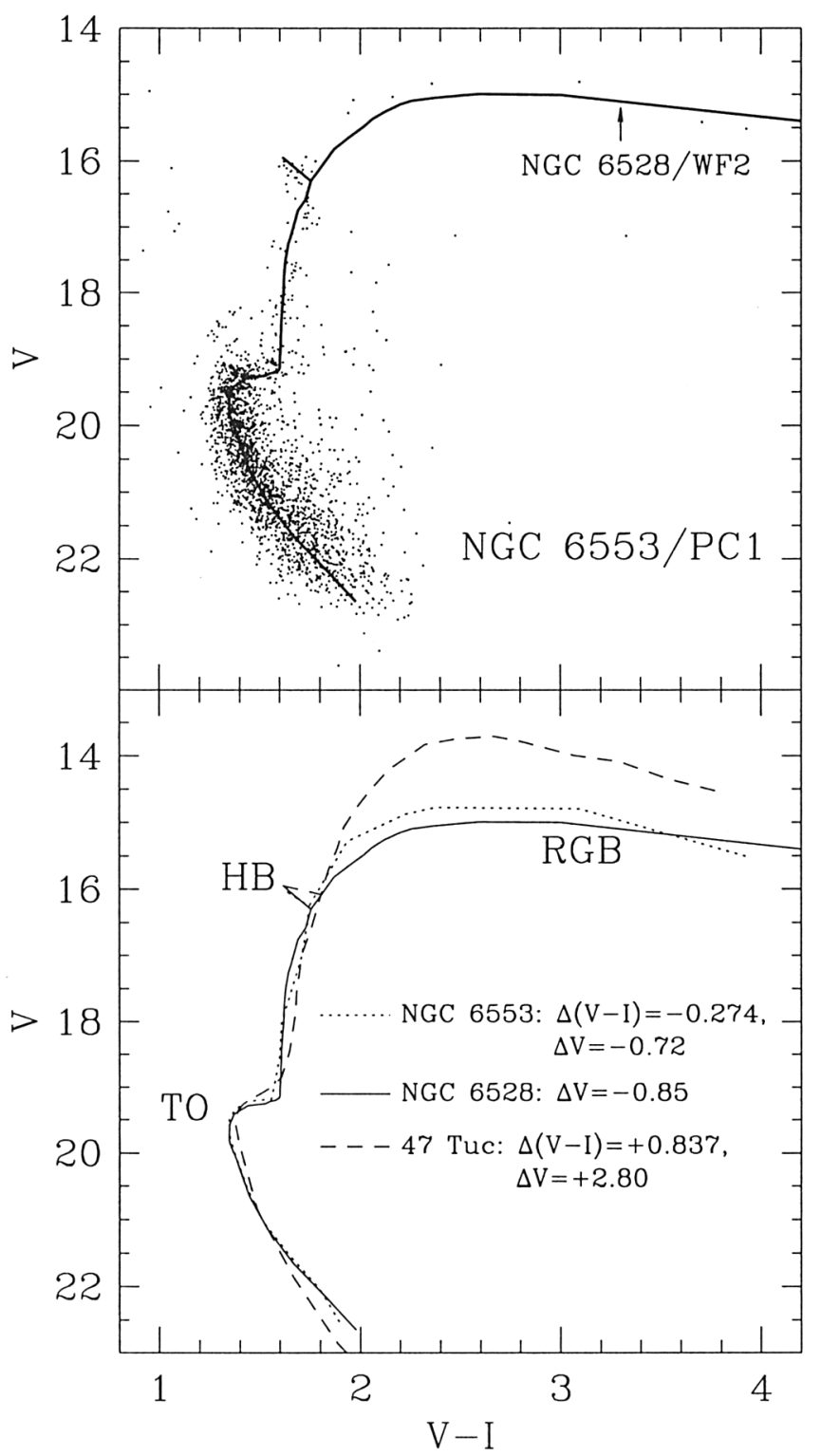

Figure 2. The CMD of the metal rich globular cluster NGC 6553, obtained using WFPC2 V,I imagery (HST - GO 5963) by S. Ortolani et al. (1995); overplotted on these data is the locus of NGC 6528, in near perfect agreement. In the lower panel of the figure, the mean locus of NGC 6528 is plotted along with those of NGC 6553 and the wellknown inner halo globular cluster 47 Tuc. Notice how closely $\Delta V_{T O}^{H B}$ agrees between the 3 clusters. Note that the age is well defined by the difference in luminosity between the horizontal branch and the turnoff. 
clear therefore that the overall metallicity of $\mathrm{NGC} 6553$ is around $\left[\mathrm{Z} / \mathrm{Z}_{\odot}\right]=-0.2$ to solar. This is compatible with the bending of the RGB of NGC 6553/NGC 6528 relative to 47 Tuc as can be seen in Fig. 2 .

In Fig. 3 we show VLT-UVES spectra of the [O I] $76300.311 \AA$ oxygen line of a giant in NGC 6528 and that of EROS-BLG-2000-5, a field bulge giant observed through microlensing magnification. Our analysis in collaboration with other authors (in preparation) shows that oxygen-to-iron is overabundant in both stars.

Table 1. Abundances of individual stars of NGC 6553 from Barbuy et al. (1999) for red giants and Cohen et al. (1999) for horizontal branch stars, compared to mean values found for the sample of field Baade's Window stars by McWilliam \& Rich (1994).

\begin{tabular}{crrr}
\hline$[\mathrm{X} / \mathrm{Fe}]$ & NGC6553:Barbuy & NGC6553:Cohen & MR94 \\
\hline$[\mathrm{Fe} / \mathrm{H}]$ & -0.55 & -0.17 & -0.25 \\
$\mathrm{Na}$ & +0.65 & - & +0.20 \\
$\mathrm{O}$ & $(0.3)$ & $+0.5 / 0.0$ & +0.03 \\
$\mathrm{Mg}$ & +0.33 & +0.40 & +0.35 \\
$\mathrm{Al}$ & +0.50 & +0.36 & +0.58 \\
$\mathrm{Si}$ & +0.35 & +0.14 & +0.14 \\
$\mathrm{Ca}$ & +0.32 & +0.26 & +0.14 \\
$\mathrm{Ti}$ & +0.60 & +0.20 & +0.37 \\
\hline $\mathrm{Z}$ & 0.009 & $0.025 / 0.013$ & 0.013 \\
{$\left[\mathrm{Z} / \mathrm{Z}_{\odot}\right]$} & -0.27 & $+0.16 /-0.12$ & -0.12 \\
\hline \hline
\end{tabular}

Table 2. Values of $[\mathrm{Fe} / \mathrm{H}],[\mathrm{O} / \mathrm{Fe}],[\alpha / \mathrm{Fe}]$ and corresponding $\left[\mathrm{Z} / \mathrm{Z}_{\odot}\right]$

\begin{tabular}{crrr}
\hline$[\mathrm{Fe} / \mathrm{H}]$ & {$[\mathrm{O} / \mathrm{Fe}]$} & {$[\alpha / \mathrm{Fe}]$} & {$\left[\mathrm{Z} / \mathrm{Z}_{\odot}\right]$} \\
\hline-0.5 & +0.30 & 0.00 & -0.32 \\
-0.5 & +0.30 & +0.30 & -0.26 \\
-0.5 & +0.40 & 0.00 & -0.25 \\
-0.5 & +0.40 & +0.40 & -0.18 \\
-0.5 & +0.50 & +0.50 & -0.09 \\
\hline \hline
\end{tabular}

\section{Conclusions}

The study of bulge globular clusters has made considerable progress in the last decade, thanks to CCD and IR detectors combined to telescopes with much 
improved performances in terms of spatial resolution such as the HST and other new generation telescopes. The structure of the bulge is now better known, given the more accurate distances, metallicities and horizontal branch types derived based on well defined CMDs. The metal-rich clusters show a strong concentration around the Galactic center and define a bulge structure rather than a thick disk distribution according to Minniti (1995) and Côté (1999). The metal-poor clusters in the inner parts of the Galaxy may partly belong to the halo, but some of them might be true bulge members, in which case they might be the oldest objects in the Galaxy - such studies remain to be done. As to whether the Galactic bulge is old, or is the result of a dynamical evolution of the bar, there are evidences for both considerations: the bulk of the Galactic bulge seems to be old, but there is a younger component, so that probably there is a mixture of stellar populations. Finally, metallicity and abundance ratio determinations are presently available for very few stars. In particular, it is important to analyse stars in clusters ranging from the red giant tip to the horizontal branch, since these stars show a broad range of temperatures and gravities, and effects of model atmospheres may affect results if different kinds of stars are analysed. Results should be much improved with new generation $8 \mathrm{~m}$ telescopes equipped with high resolution optical and infrared spectrographs.

\section{References}

Amaral L.H., Ortiz R., Lépine J., Maciel W.J. 1996, MNRAS, 281, 339

Barbuy B., Bica E., Ortolani S. 1998, A\&A, 333, 117

Barbuy B., Ortolani S., Bica E., Desidera S. 1999, A\&A, 348, 783

Barbuy B., Renzini A., Ortolani S., Bica E., Guarnieri M.D., 1999, A\&A, 341, 539

Bica E. 1988, A\&A, 195, 76

Burton W.B., Liszt H.S. 1983, A\&AS, 52, 63

Cassisi S. and Salaris M. 1997, MNRAS, 285, 593

Cassisi S., Castellani V., Degl'Innocenti S., Weiss A. 1998, A\&AS, 129, 267

Coelho P., Barbuy B., Perrin M.-N., Idiart T., Schiavon R.P., Ortolani S., Bica E., 2001, A\&A, 376, 136

Cohen J.G., Gratton R.G., Behr B., Carretta E., 1999, ApJ 523, 739

Côté P., 1999, AJ 406, 1999

Da Costa G. 1995, PASP, 107, 937

Friedli D., Benz W. 1995, A\&A, 301, 649

Gadotti D., dos Anjos S. 2001, AJ, 122, 1298

Harris W.E. 1996, AJ, 112, 1487

Harris W.E., Phelps R.L., Madore B.F. et al. 1997, AJ, 113, 688

Hurt R.L., Jarrett T.H., Kirkpatrick J.D. et al. 2000, AJ, 120, 1876

Irwin M.J., Demers S., Kunkel W.E. 1995, ApJ, 453, L21

Larson R.B. 1990, PASP, 102, 709

McWilliam A., Rich R.M. 1994, ApJS, 74, 1075 


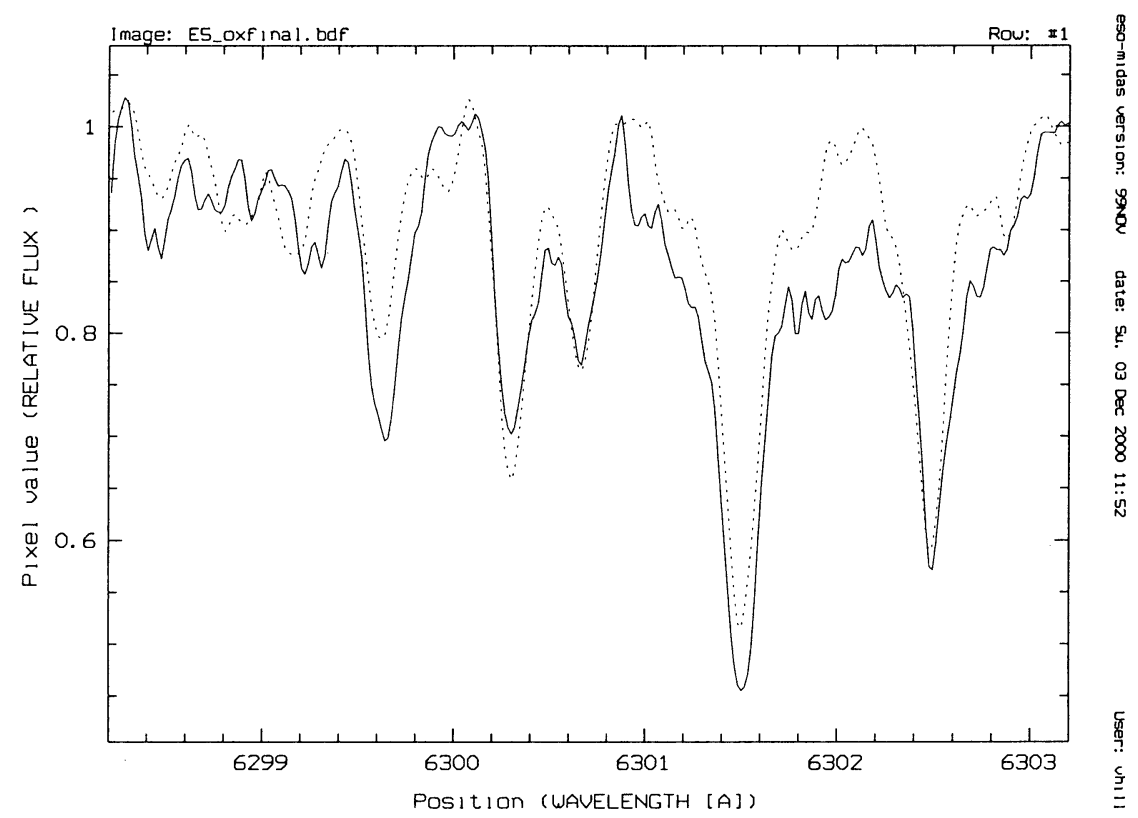

Figure 3. VLT-UVES spectra of a giant star in NGC 6528 (solid line) and EROS-BLG-2000-5, a field bulge star observed through microlensing magnification. The region is chosen to show the oxygen line $[\mathrm{O} \mathrm{I}] \lambda$ $6300.311 \AA$.

Minniti D. 1994, PASP, 106, 813

Minniti D. 1995, AJ, 109, 1663

Norman C.A., Sellwood J.A., Hasan H. 1996, ApJ, 462, 114

Ortolani S., Barbuy B., Bica E., Renzini A., Zoccali M., Rich R.M., Cassisi S. 2001, A\&A, submitted

Ortolani S., Bica E., Barbuy B. 1993, A\&A, 273, 415

Ortolani, S., Bica, E., Barbuy, B. 2000, A\&A, 361, L57

Ortolani S., Renzini A., Gilmozzi R., Marconi G., Barbuy E., Bica E., Rich R.M. 1995, Nature, 377, 701

Raha N., Sellwood J.A., James R.A., Kahn F.D. 1991, Nature, 352, 411

Rich R.M., McWilliam A. 2000, SPIE, 4005, 150

Sarajedini A., Geisler D. 1996, AJ, 112, 2013

Tavarez M., Friel E.D. 1995, AJ, 110, 223

Terndrup D.M. 1988, AJ, 96, 884

Van den Bergh S. 1993, ApJ, 411, 178

Weinberger R. 1995, PASP, 107, 58

Zoccali M., Renzini A., Ortolani S., Bica E., Barbuy B., 2001, AJ, 121, 2638 


\section{Discussion}

K. Olsen: Is there spatial coherence in the $\mathrm{E}(\mathrm{B}-\mathrm{V})$ map for the differentially reddened cluster NGC 6553 ? Are the bulge clusters typically embedded in the dust or is it foreground reddening?

B. Barbuy: A plot of $\mathrm{E}(\mathrm{B}-\mathrm{V})$ vs. cluster distance for the inner clusters was shown in Barbuy et al. (1998), where it was clear that there is no correlation between these quantities, suggesting that the dust is foreground to the clusters, i.e. close to us. As for NGC 6553 there is a differential reddening of about $\triangle E(B-V)=$ 0.2 , which is patchy rather than 'well behaved'. It would be important to do a detailed study of the reddening along the cluster area, in particular in view of deriving stellar effective temperatures. 